\title{
Las necesidades de las adolescentes madres en el contexto educativo: un estudio de caso en una institución de educación pública ${ }^{1}$
}

Daniel Martínez Esquivel ${ }^{2}$, Jennifer Chaves Guillén ${ }^{3}$, María Jacinta Guzmán Rojas ${ }^{4}$, Luis Martínez López ${ }^{5}$, Damián Meneses ${ }^{6}$, Rebeca Mora Martínez ${ }^{7}$, Nazira Quesada Mora ${ }^{8}$, Sofía Vargas Quesada ${ }^{9}$

Institución: Universidad de Costa Rica

\section{COMO CITAR}

Martínez, D. (2015). Las necesidades de las adolescentes madres en el contexto educativo: un estudio de caso en una institución de educación pública. Rev. Enfermería Actual en Costa Rica, 28, 1-16. DOI: http://dx.doi.org/10.15517/revenf.v0i28.17059

\section{RESUMEN}

Introducción. El embarazo en la adolescencia es un problema de salud pública que debe ser subsanado en las generaciones presentes a partir de la educación en salud. La Enfermería no debe mostrarse ajena a este proceso, sino que debe ser sensible a las necesidades tanto de las adolescentes en riesgo como de las adolescentes embarazadas, motivo por el que en esta investigación se planteó como pregunta ¿cuáles son las necesidades surgidas en el ámbito educativo en las adolescentes embarazadas?

Método. Se diseñó un estudio cualitativo, fenomenológico. Los datos fueron recolectados mediante la técnica de grupo focal en el que participaron tres adolescentes madres. El análisis se realizó desde una perspectiva interpretativa.

Resultados. Los principales resultados evidenciaron necesidades personales como uniformes que se ajusten a sus cambios físicos, necesidades de infraestructura como sillas y pupitres adecuados, un espacio específico para la lactancia, tiempo para ver a su hijo o hija y adecuación de los horarios de clase.

Conclusión. Se concluye que la implementación de las políticas públicas favorecerá el respeto de los derechos de las adolescentes madres en aspectos personales, académicos y de infraestructura.

Palabras clave: adolescencia, embarazo-adolescente, enfermería, necesidades-de-salud, salud.

\footnotetext{
${ }^{1}$ Fecha de aceptación: 20 de octubre del 2014

Fecha de recepción: 2 de setiembre del 2014

${ }^{2}$ Universidad de Costa Rica. Escuela de Enfermería. Costa Rica. Correo electrónico: dtinez@gmail.com

${ }^{3}$ Estudiante de Licenciatura en Enfermería. Universidad de Costa Rica. Costa Rica. Correo electrónico: jcguillen1989@yahoo.com

${ }^{4}$ Estudiante de Licenciatura en Enfermería. Universidad de Costa Rica. Costa Rica. Correo electrónico: majacigr@yahoo.com

${ }^{5}$ Estudiante de Licenciatura en Enfermería. Universidad de Costa Rica. Costa Rica. Correo electrónico: 1uis.tico88@gmail.com

${ }^{6}$ Estudiante de Licenciatura en Enfermería. Universidad de Costa Rica. Costa Rica. Correo electrónico: damianmb08@gmail.com

${ }^{7}$ Estudiante de Licenciatura en Enfermería. Universidad de Costa Rica. Costa Rica. Correo electrónico: rebe1692@hotmail.com

${ }^{8}$ Estudiante de Licenciatura en Enfermería. Universidad de Costa Rica. Costa Rica. Correo electrónico: crigo0612@hotmail.com

${ }^{9}$ Estudiante de Licenciatura en Enfermería. Universidad de Costa Rica. Costa Rica. Correo electrónico: ansofvar91@hotmail.com
} 


\title{
The needs of teenage mothers in the educational context: a case study in an institution of public education ${ }^{1}$
}

Daniel Martínez Esquivel ${ }^{2}$, Jennifer Chaves Guillén ${ }^{3}$, María Jacinta Guzmán Rojas ${ }^{4}$, Luis Martínez López ${ }^{5}$, Damián Meneses $^{6}$, Rebeca Mora Martínez ${ }^{7}$, Nazira Quesada Mora ${ }^{8}$, Sofía Vargas Quesada ${ }^{9}$

Institution: University of Costa Rica

\section{CITED:}

Martínez, D. (2015). The needs of teenage mothers in the educational context: a case study in an institution of public education. Rev. Enfermería Actual en Costa Rica, 28, 1-26. DOI: http://dx.doi.org/10.15517/revenf.v0i28.17059

\begin{abstract}
Introduction. The teenage pregnancy is a public health problem that should be remedied in the present generation from health education. Nursing cannot ignore this process but must be sensitive to the needs of at-risk adolescents and pregnant teenagers. Therefore, this research was to answer what are the needs arising in the field of education in pregnant teens?

Method. We designed a qualitative, phenomenological study. Data were collected by the technique of focus group involving three teenage mothers. The analysis was carried out from an interpretive perspective.

Results. The main results showed personal needs like uniforms that meet their physical, infrastructure needs such as adequate chairs and desks, a specific space for breastfeeding, time to see your child and adequacy of class schedules.

Conclusion. It is concluded that the implementation of public policies promotes respect for the rights of teenage mothers in personal, academic and infrastructural aspects.

Key words: adolescence, health, health-needs, nursing, teen-pregnancy.

\footnotetext{
${ }^{1}$ Date of acceptance: October 20, 2014

Date of receipt: September 2, 2014

${ }^{2}$ Undergraduate Nursing. University of Costa Rica. School of Nursing. Costa Rica. E-mail: dtinez@gmail.com

${ }^{3}$ Undergraduate Nursing. University of Costa Rica. School of Nursing. Costa Rica. E-mail: jcguillen1989@yahoo.com

${ }^{4}$ Undergraduate Nursing. University of Costa Rica. School of Nursing. Costa Rica. E-mail: majacigr@yahoo.com

${ }^{5}$ Undergraduate Nursing. University of Costa Rica. School of Nursing. Costa Rica. E-mail: luis.tico88@gmail.com

${ }^{6}$ Undergraduate Nursing. University of Costa Rica. School of Nursing. Costa Rica. E-mail: damianmb08@gmail.com

${ }^{7}$ Undergraduate Nursing. University of Costa Rica. School of Nursing. Costa Rica. E-mail: rebe1692@hotmail.com

${ }^{8}$ Undergraduate Nursing. University of Costa Rica. School of Nursing. Costa Rica. E-mail: crigo0612@hotmail.com

${ }^{9}$ Undergraduate Nursing. University of Costa Rica. School of Nursing. Costa Rica. E-mail: ansofvar91@hotmail.com
} 


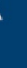

\section{INTRODUCCIÓN}

Según la Organización Mundial de la Salud (OMS, 2013), el período que comprende la adolescencia comienza a los diez años, pero esta etapa no se rige por límites etarios, ni se apoya sobre bases estrictas para dar un veredicto de que ha finalizado; al respecto, Krauskopf (1999) menciona que es más que una construcción social del individuo y una compleja búsqueda de la identidad, acerca de lo que se puede afirmar que, contrario a la descripción de las cifras etarias que emite la OMS (2013), la etapa de la adolescencia es subjetiva para cada joven, durante la que los límites establecidos fungen como un marco relativo para establecer el inicio y el final de la etapa.

Dado lo anterior, resulta complicado establecer un rango preciso para definir cuándo termina esta fase, caracterizada por cambios a nivel físico, psicológico y social, cuyo significado es inmenso y determinante para cada persona (García y Deval, 2010). La adolescencia no está enmarcada en una línea del tiempo específica, sino que es un proceso lleno de complejidad que, desde sus inicios, implica cambios a nivel general a través de los que se madura el pensamiento hasta dar por un hecho que se está en el momento final de la adolescencia.

Este proceso, el de ser adolescente, incluye un componente histórico con un gran número de significados sociales y lingüísticos que influyen en la construcción del individuo y de su comportamiento ante la sociedad (Quesada, Montero, Prieto y Rodríguez, 2010). Conforme se dan esos cambios en el pensamiento, en lo que respecta al entorno y al ámbito físico, van apareciendo los desafíos, los cuales se apoderan de la rutina de cada adolescente: las dudas comienzan a surgir, a levantarse desde lo más interno de cada ser para salir como gritos silenciosos ante una sociedad que parece, en muchas ocasiones, la fuente primaria que esclarecerá esas cuestiones (Páramo, 2009). Entonces, la adolescencia no se restringe a festejos en el hogar, a reuniones de café y pastel con amistades o a actividades comunitarias en pro de la ecología, va más allá, puesto que se incluye en prácticas sociales más liberales, como el inicio temprano de la actividad sexual coital, del consumo de sustancias nocivas para la salud integral de la persona, el vivir fiestas desenfrenadas, entre otras conductas riesgosas (Soutullo y Mardomingo, 2010).

Esta inmersión en actividades riesgosas tiene consecuencias que muchas veces marcan fuertemente esta etapa, al punto de que muchos jóvenes se aproximan de manera prematura con una realidad que, al parecer, no estaba pensada dentro de su protocolo de vida. El ejemplo más claro lo revelan las estadísticas de Costa Rica en cuanto a embarazos en adolescentes, dato mencionado por Anchía, Badilla, Cordero y Marín (2012) y obtenido a través del Instituto Nacional de Censos y Estadísticas (INEC), entidad que afirma que "en el año 2011, se reportaron 13867 partos de mujeres menores de 19 años, lo cual corresponde al $18 \%$ del total de partos reportados a nivel nacional, cifra que ha variado poco desde 1998" (p.1).

Algo grave que se debe destacar es que el $80 \%$ de estos embarazos son mediados por hombres que llevan una enorme ventaja respecto a la edad en comparación con estas madres adolescentes (Contreras, 2013); aunque la cifra es preocupante, lo es más la causa de los embarazos en estas jóvenes: en muchos casos se habla de hombres cuya edad ronda los 40 años, quienes establecen una relación de poder que aumenta la vulnerabilidad de las adolescentes, todo amparado en una construcción de género en la que el patriarcado observa con normalidad este tipo de conductas. 


\section{.}

Respecto de este panorama, hay que mencionar la situación de las regiones Brunca y Atlántica del país, donde más se reporta embarazos adolescentes. En cuanto a la gran área metropolitana, en San José se presentan mayor cantidad de embarazos, principalmente hacia el sur del Valle Central, en Alajuelita: ambas son las áreas de mayor incidencia y prevalencia en el país (Contreras, 2013). Estas ciudades nacionales reflejan una realidad que describe cómo los sectores que presentan un alto problema de vulnerabilidad social, se encuentran entre los lugares donde la problemática se acrecienta, mas no por eso se debe obviar la presencia de dicha problemática social en el resto del país y reforzarse las medidas preventivas, dado que el número de madres adolescentes sigue en aumento, así como la deserción de los centros educativos.

El escenario actual pone en riesgo a gran número de adolescentes, quienes deben padecer las consecuencias de un embarazo en la edad adolescente, el cual no solo obstaculiza la consecución del proyecto de vida, sino que genera condiciones que, desde las diversas aristas (social, económica, educativa), complican el panorama a futuro de la madre adolescente y el nuevo ser. En relación con lo anterior, Zamora (2011), menciona que los embarazos de adolescentes reflejan un fenómeno con repercusiones a nivel social que afectan el ámbito educativo, al punto de que se convierte en un problema de salud pública, debido a que

Se encuentran vinculados a problemas de pobreza, educación, inequidad de género, vulneración de los derechos y contextos familiares adversos. No es un hecho aislado, sino determinado por un conjunto de variables macro y micro sociales que inciden fuertemente en su ocurrencia (p.19).

Tal situación trae consigo una gran cantidad de retos, puesto que las introduce, muchas veces, en un mundo de desventajas a nivel educativo y de género, que limita, entre otros rubros, las oportunidades a futuro que suele desembocar en entornos de de pobreza de estas madres y sus familias, de modo que aumentan su vulnerabilidad, así como la estratificación de las comunidades (Quesada et al, 2010). Pero dentro de todo este difícil contexto, las prácticas sexuales tempranas y riesgosas son apenas la punta del problema, el cual surge en un ambiente en el que los jóvenes se exponen a otras problemáticas sociales en las que son víctimas de las diferencias o inequidades que surgen en las diversas sociedades humanas.

Con lo mencionado se quiere dar a entender la significativa suma de causas que acompañan cada embarazo, las tareas maternas y paternas que llegan en un período durante el cual no se está lo suficientemente preparado o preparada para llevarlas de la manera óptima, y que generan cambios fuertes en las condiciones biopsicosociales de los actores presentes en dicha situación (López, 2011), siempre considerando que la problemática va más allá de una simple decisión.

En relación con el tema, es primordial referirse al entorno en el cual se desenvuelven estos jóvenes. En los embarazos en adolescentes, no se trata de haber olvidado el preservativo, de desatender lo que decía el padre o la madre acerca de cómo cuidarse o simplemente esperar a tener más edad, ni tampoco el asunto versaba sobre la pena que sentían cuando la duda se asomaba o a seguir el patrón de facilidad como susurraba el vecindario: es más que eso, puesto que "los determinantes sociales se relacionan con la existencia de embarazo en la adolescencia, de lo que se ha observado que cuanto mayor es la relación que ejercen, así será la proporción en cuanto a la cantidad de embarazos en edades tempranas de la vida"(Barrientos y Tobar, 2011, p. 146). 
Dicha afirmación confirma la idea de que los factores sociales son generadores primarios de la problemática de embarazos en la adolescencia, producto de un sistema que genera condiciones de vulnerabilidad y exclusión social a una buena parte de las poblaciones, lo cual aumenta la incidencia de casos entre los sectores donde la situación social, económica y educativa difiere de manera negativa.

El embarazo genera cambios en la madre, en su pareja o en todo el núcleo familiar que los rodea, en el ámbito físico, psicológico, social y económico; y se maximizan cuando la nueva vida que se encuentra en desarrollo proviene de un vientre adolescente, debido a que en esta etapa la gestación es observada como un problema médico ya sea por los riesgos en el embarazo y el parto y por las implicaciones sociales y psicológicas que tendrán un marcado costo a nivel personal, social, educacional y familiar (Molina, Ferrada, Pérez, Cid, Casanueva y García, 2004).

Estos cambios y riesgos potenciales que acompañan al embarazo precoz representan un reto para indagar acerca de las necesidades que presentan las madres adolescentes, ya sea que estén inmersas en su hogar, en la comunidad, en el ámbito educativo o en cualquier espacio al que pertenezcan. En relación con lo anterior, Noguera y Alvarado (2012) mencionan que cada joven madre podría experimentar rechazo, temor y mucha confusión durante esta etapa, debido a que las dificultades afloran y las soluciones muestran gruesas espaldas; por consiguiente, es fundamental la presencia de la Enfermería, como compañía diaria y planificando en conjunto las soluciones efectivas con las mejores estrategias en las cuales se plasme el conocimiento enfermero, con el fin de mejorar la calidad de vida de estas mujeres y sus sistemas personal, interpersonal y social.

En cuanto a su quehacer, Redondo, Galdo y García (2008) consideran que el equipo de salud tiene como objetivo ayudar a la adolescente a aceptar su embarazo, fortalecer los vínculos familiares y brindar atención perinatal integral, funciones que se asemejan a las que cada profesional dentro de lo la Enfermería debe fomentar en su práctica e intervención diarias. Ese acompañamiento es coherente con los planteamientos de la Organización Panamericana de la Salud (OPS, 2008), en cuanto a necesidad de desarrollar tácticas que garanticen el máximo nivel de salud posible de cada una de las personas dentro de un sistema de salud; en este caso y de manera individual se procurará brindar una atención integral e integrada a la adolescente madre. La intervención por parte del equipo de salud se adecua a las necesidades de esta y se proporciona de una manera holística en la que se le invita a participar en el mismo proceso.

Una vez detectado lo anterior, se pretendió responder a la pregunta, ¿cuáles son las necesidades surgidas en el ámbito educativo en las adolescentes embarazadas, que cursan la secundaria en el Liceo Roberto Brenes Mesén, ubicado en Hatillo 2, durante el segundo semestre del año 2013?

\section{MATERIALES Y MÉTODO}

La presente investigación es cualitativa, la cual según Hernández, Fernández y Baptista (2010), consiste en un método de investigación en el que se recolecta y analiza los datos sin medición numérica para responder a las preguntas que surjan en el proceso de interpretación. El objetivo de este tipo de estudio es conocer la perspectiva de los participantes acerca de los fenómenos y situaciones que los rodean para así poder profundizar en sus experiencias, perspectivas, y opiniones. 
El diseño del trabajo fue fenomenológico que, según Báez y Tudela (2009), presta atención a la realidad actual, denominada fenómeno. Según Hernández et al (2010), la perspectiva del enfoque fenomenológico busca analizar los valores y ritos de un determinado grupo social que, en este caso, se trata del embarazo adolescente y sus necesidades dentro del contexto educativo.

Para la presente investigación, se eligió siete mujeres embarazadas o en periodo de lactancia no mayor a seis meses, de las cuales solo tres participaron. Los criterios de inclusión considerados fueron: que sean mujeres entre los 13 y 17 años, que se encuentren embarazadas o en periodo de lactancia no mayor a seis meses y que estudien en el Liceo Roberto Brenes Mesén en el año 2013.

El método elegido para obtener la información fue grupo focal que, según Hernández et al. (2010). "consiste en reuniones de grupos pequeños en donde los participantes conversan en torno a uno o varios temas en un ambiente relajado e informal, bajo la conducción de un especialista en dinámicas grupales" (p. 425). En este formato se reúne a un grupo de personas y se trabaja en relación con los conceptos, las experiencias, las emociones, los sucesos o los temas que interesan en el planteamiento de la investigación. Se realizó una guía de preguntas que fue validada por tres expertas de la Escuela de Enfermería de la Universidad de Costa Rica.

Las preguntas planteadas al grupo focal fueron:

1. ¿Considera que ha tenido suficiente apoyo durante el proceso de embarazo o posterior a este?

2. ¿Cuáles considera que son sus necesidades para desenvolverse como adolescente madre dentro de la institución?

3. ¿Qué oportunidades le gustaría tener en el colegio?

4. ¿Cuáles son sus planes, objetivos o metas en la vida?

De estas preguntas se derivaron las siguientes unidades de análisis:

1. Apoyo social

2. Necesidades y oportunidades educativas

3. Proyecto de vida

A partir de la información obtenida, se efectuó un análisis interpretativo mediante una triangulación de datos; en la indagación cualitativa se posee una mayor riqueza, amplitud y profundidad en los datos si estos provienen de diferentes actores del proceso, de distintas fuentes y si se utiliza una mayor variedad de formas de recolección de los datos; a ese proceso, en el que se busca diferentes fuentes y métodos de recolección, se le denomina triangulación de datos (Hernández et al, 2010). Con la ayuda de la triangulación, se conjugaron los fundamentos teóricos con la posición de quienes investigan y las respuestas obtenidas en el grupo focal, de quienes se obtuvo lo necesario el análisis que respondió a la interrogante de la investigación.

\section{Consideraciones éticas}

Las consideraciones éticas que se tomaron en cuenta pertenecen a la Declaración Universal sobre Bioética y Derechos Humanos de la Organización de las Naciones Unidas para la Educación, la Ciencia y la Cultura, UNESCO por sus siglas en inglés (2005), de la cual se incluyó las siguientes: 
Se respetó los derechos humanos, como la libertad, y la dignidad de las adolescentes, de igual modo se priorizó el bienestar de las adolescentes por encima de la investigación.

Las adolescentes tuvieron acceso al consentimiento informado en el que se explica cuál es el propósito de la investigación, los riesgos de participar, así como los beneficios para ellas y para la comunidad científica. Las adolescentes tenían libertad de negarse a participar o retirarse en cualquier momento de la investigación, si así lo decidían.

Por encima del conocimiento que se pretendía obtener, se respetó y protegió la integridad de las adolescentes participantes como seres humanos de derecho y población vulnerable.

Las adolescentes fueron notificadas de que la información brindada sería confidencial, para el uso exclusivo de la investigación descrita y por los y las estudiantes participantes únicamente.

Por ningún motivo se perpetró actos de discriminación o pensamientos que estigmatizaran a las adolescentes participantes del proyecto.

Por último, se les indicó a las adolescentes que dicho trabajo y las aplicaciones que generara iban a ser para el beneficio no solo del personal de Enfermería, sino también para ellas como adolescentes madres.

\section{RESULTADOS}

La sociedad produce un gran impacto sobre las adolescentes embarazadas y, de manera recíproca, estas jóvenes impactan el entorno social que les rodea: la sociedad interacciona con ellas a través de políticas, leyes y oportunidades que genera para esta población. De igual modo, determina el acceso o las limitaciones que poseerán respecto de satisfacer las necesidades que tengan, en otras palabras, cuando las acciones que propician la mejora en la calidad de vida adaptada al estado de embarazo de estas adolescentes trasciende más allá de lo proclamado, las oportunidades se acercan más a cada una de ellas.

De acuerdo con la información recolectada, se identificó las necesidades de las adolescentes madres en el ámbito educativo las cuales se presentan a continuación, siguiendo cada unidad de análisis propuesta.

\section{Unidad de análisis: apoyo social}

En cuanto al apoyo social, por un lado, las participantes se refirieron primero al Estado costarricense como fuente de recursos económicos, sin embargo, aunque el gobierno cuente con instituciones para brindar este tipo de apoyo, al parecer no es suficiente para toda la población que lo requiere. Así lo menciona una de las participantes cuando afirma que “me dijeron que no había presupuesto, por eso tampoco podían ayudarnos" (Grupo focal, 2013).

Por otro lado, las participantes identificaron a su familia como la principal fuente de apoyo. Una de las respuestas obtenidas fue "mi mamá, o sea, es como muy dulce y siempre está preguntándome y está diciéndome que qué quiere, que qué ocupa, entonces siempre la tengo ahí y de parte del muchacho también” (Grupo focal, 
2013). La respuesta de la adolescente madre evidencia que cuenta con el apoyo de su familia, particularmente el de su madre.

También, se refirió al apoyo recibido por parte de su pareja o el padre de su hijo o hija, al responder "él me ayuda con las citas, es él el que me recuerda... a mí todo se me va. Entonces, él me recuerda: ¿pasó por las pastillas? Y yo: uy se me olvidaron. Entonces, ya va él o me da plata para ir yo o así” (Grupo focal, 2013),lo cual confirma el apoyo que existe por parte de la pareja. Aunque en algunos casos viven juntos y en otros no, todas coincidieron en que los progenitores están pendientes de todo lo referente al embarazo y a sus necesidades.

Además, se evidenció que cuentan con el apoyo de sus familia desde siempre y que inclusive éste aumentó, en algunos casos, en el momento en que estas les comunicaron sobre su embarazo, “...desde el principio yo sentí el apoyo pero aumentó conforme avanzó el embarazo” (Grupo focal, 2013).

Unidad de análisis: necesidades y oportunidades educativas

Al consultar sobre las necesidades y oportunidades educativas, todas respondieron "sillas cómodas" (Grupo focal, 2013), lo cual representa una limitante de infraestructura que no se ha tomado en cuenta en el centro educativo.

Otra necesidad que mencionaron fue la de un espacio físico para la lactancia, cuando una de ellas dijo “diay, así como un lugar donde nos permitan dar lactancia" (Grupo focal, 2013).

También se mencionó el uniforme: “no mucho, bueno, primero por lo de las camisas. Hay muchos problemas. Llega el momento en que la camisa no te da, o la camisa queda apretada, entonces, ya ahí uno empieza a venir con otras blusas (Grupo focal, 2013). Al igual que la infraestructura, la vestimenta puede afectar el bienestar o la comodidad de la madre adolescente que, en este caso, debe ser provisto por la institución.

En cuanto al lazo entre madre e infante, una joven comentó: “Por lo menos tiempo, para ir a verlo" (Grupo focal, 2013). La promoción del apego madre e hijo siempre ha sido un punto importante de intervención para la Enfermería, ya que beneficia el desarrollo de los lazos maternos y el propio desarrollo del bebé

Por último, las participantes mencionaron, “también que a uno le puedan acomodar el horario" (Grupo focal, 2013), consideración que refleja la necesidad de la protección de los derechos tanto de las madres como de sus hijos para mejorar sus oportunidades de calidad de vida.

Unidad de análisis: proyecto de vida

Al consultar sobre sus objetivos y metas, las adolescentes madres mostraron interesadas en superarse , como expresa una de las participantes “de hecho yo mínimo seguiría estudiando” (Grupo focal, 2013). Ante este panorama, es necesario implementar o promover más oportunidades en el país. 


\section{.}

\section{DISCUSIÓN}

El impacto de la sociedad en la vida de la adolescente madre alude a que el embarazo durante esta etapa vital no solamente tiene implicaciones individuales y familiares sino sociales. El entorno social debe brindar las mejores condiciones posibles para la adolescente madre y el niño o niña por nacer ya que, la madre, no solamente enfrenta el peso de llevar la carga propia de la adolescencia como etapa, sino de todas las repercusiones que este tipo de embarazos conlleva.

La sociedad se maneja entre aspectos positivos y negativos que influyen sobre las madres desde lo que sus acciones reflejen o produzcan como resultado, por ello la sociedad debe velar que toda acción y decisión sea beneficiosa y proteja a la adolescente madre y al futuro infante, tarea en la que se precisa la implementación de políticas públicas, la inversión del gobierno y el total compromiso para que las acciones sean realmente satisfactorias.

Con el fin de complementar lo anteriormente descrito, en la Política Nacional para la Niñez y Adolescencia (2009), se menciona que "el Estado se compromete a destinar el máximo de los recursos disponibles para ejecutar planes nacionales, programas, proyectos y actividades dirigidos a la garantía de los derechos de las personas menores de edad" (p. 64), lo cual concuerda con el interés que esta investigación en cuanto a las necesidades que expresan las adolescentes madres en estudio y que de una manera $u$ otra no han sido satisfechas.

Una sociedad basada en la equidad debe brindar las mismas oportunidades a sus integrantes sin importar grupo etario, condición económica o social o si se es adolescente madre o no; es decir, el Estado debe procurar que cada uno de sus habitantes disfrute de los mismos derechos, de modo que se rompa toda brecha que impida a ciertos grupos poblacionales, como en este caso las adolescentes madres, gozar de igualdad de oportunidades. Como parte de este esfuerzo, en el país se busca promover y alcanzar la equidad social a través de la implementación de políticas públicas, así como fomentar la participación del gobierno como ente protector de la ciudadanía, incluyendo sin duda alguna a este grupo de madres.

El fortalecimiento de los derechos y oportunidades que se les brinde a las adolescentes madres es capaz de mejorar la calidad de vida, dado que se procura hacer valer aspectos de salud, educación, cultura y, en sí, del desarrollo humano en general. Al respecto, la Política Nacional para la Niñez y Adolescencia (2009) defiende que

La niñez y la adolescencia tendrán un trato prioritario en la lucha contra la pobreza y la exclusión social, que deberá asumir sus entornos familiares y comunitarios, desde una perspectiva integral, intersectorial e interinstitucional. Todo con miras a la creación de la igualdad de oportunidades para la procura de su bienestar y desarrollo, y la facilitación del acceso a la oferta programática de la red de protección social del Estado y la sociedad costarricense, con la combinación de instrumentos asociados a las políticas sociales universales y los de la política social selectiva (p. 63).

Sin embargo, la acción del Estado ha sido limitada por diferentes razones que han generado inequidades en salud dentro de las adolescentes madres, cuya consecuencia es el empobrecimiento de su calidad de vida en diferentes ámbitos como el educativo. Dicha situación desencadena problemáticas que limitan las oportunidades 
de estudio de las madres, quienes se ven obligadas a desertar, lo cual estanca su nivel educativo y se convierte en un obstáculo para concretar oportunidades dentro de un ámbito laboral satisfactorio que cubra las necesidades personales y genere mejores condiciones de vida para el infante que está por nacer y para los responsables más cercanos. Al respecto, el Fondo de Población de las Naciones Unidas (UNFPA, 2013) afirma que

Cuando una niña queda embarazada o tiene un hijo, su salud, educación, potencial de obtener ingresos y todo su futuro pueden estar en peligro, y puede quedar atrapada en una vida sumida en la pobreza, la exclusión y la impotencia. El efecto que sufre la madre joven se suele transmitir a su hijo, que empieza su vida con una desventaja, con lo que se perpetúa un ciclo intergeneracional de marginalización, exclusión y pobreza (p.18).

Lo anterior refuerza la idea de generar espacios apropiados para que la joven madre pueda continuar con los estudios y a su vez permanezca activo el lazo afectivo entre ella y su hijo o hija, logro que se puede obtener facilitando el acceso a los servicios o, tal como menciona el UNFPA (2013),

Asegurando el acceso a servicios para adolescentes embarazadas o madres nuevas a menudo significa proporcionar apoyo financiero para la atención de la salud y la alimentación, consejo sobre la lactancia materna, ayuda para regresar a la escuela o capacitación, refugio y servicios si sus familias las han rechazado y anticonceptivos o información (p.75).

Tal como lo propone la Política Nacional para la Niñez y Adolescencia (2009), las adolescentes madres deben hacer valer su derecho de igualdad y no discriminación, el cual exige al país que garantice a todas las personas menores de edad la protección de sus derechos y establezca políticas especiales para aquellas que se encuentran en situación de mayor vulnerabilidad; dicho programa debe promover la inclusión de todas las personas menores de edad en los programas sociales, así como su integración social, siempre en pro del respeto a la diversidad y de la aceptación de las diferencias.

Las adolescentes madres podrían lograr ese empoderamiento si establecen una comunicación asertiva con las autoridades de la institución, proceso en el que Enfermería podría servir como un canal de diálogo entre las adolescentes madres, sus familias y el personal administrativo del centro educativo, con el único objetivo de crear redes de apoyo que eviten la deserción escolar.

Además, la Enfermería debería velar por el cumplimiento de los derechos de las personas como forma de crear un empoderamiento en salud de la población, es decir, se debe dar validez a los derechos de las adolescentes con el fin de hacerlas dueñas de su salud y brindar, tal como el UNFPA (2013) indica, "un enfoque basado en los derechos humanos para el desarrollo, las personas, incluidas las mujeres y los jóvenes, no se consideran destinatarios pasivos de bienes, servicios o suministros, sino como tenedores de derechos y, por lo tanto, deben empoderarse" (p.96).

Por tales razones, se debe buscar el cumplimiento de la Política Nacional para la Niñez y Adolescencia (2009), que además propone un enfoque con vistas al desarrollo integral, el cual garantice un respeto total al derecho de las madres de continuar con su educación, elemento indispensable para su desarrollo integral, el 
asegurar que se les brinden los medios idóneos para cumplirlo, ya sea con el apoyo del patronato escolar y la junta directiva de la institución, en este caso para solventar las necesidades en cuanto a infraestructura adecuada para el embarazo.

Sin embargo, el enfoque de desarrollo integral propuesto por la misma política parece no cumplirse a cabalidad, debido a que se está limitando el acceso a la educación, entonces, si ellas no cuentan con los recursos para poder obtener la prenda adecuada y la institución no les brinda las comodidades que incluyen sillas adaptadas a su estado, podría convertirse en una razón para desertar de las aulas; es decir, se incurre en un trato desigual si se compara con el resto de la población estudiantil.

Desde este punto de vista, se puede que los determinantes de la salud, en este caso la situación económica, influyen sobre las oportunidades que puedan o no tener las personas como por ejemplo el acceso a la educación que, sin duda alguna, definirá a futuro las posibilidades de obtener una mejor calidad de vida para estas madres adolescentes. No obstante, el problema no concierne únicamente a la institución como tal, dado que es una problemática nacional, la cual radica en que no se respeta ni se le da la importancia necesaria a las políticas nacionales o, peor aún, están son divulgadas lo suficiente; ante tal situación, para la intervención de Enfermería es importante conocer y destacar estas políticas a la hora de brindar información a los adolescentes, con el objetivo de promover el empoderamiento de su situación actual de derecho, en la que la Enfermería es responsable de, según el UNFPA (2013),

Abordar el embarazo en adolescentes con protecciones de los derechos humanos ampliar el marco normativo internacional que exige a los gobiernos tomar medidas necesarias para que las niñas gocen de su derecho a la educación, a la salud y a vivir sin violencia. Los niños tienen los mismos derechos humanos que los adultos, pero también se les garantizan protecciones especiales para abordar las desigualdades inherentes a su edad. Defender los derechos, por lo tanto, puede ayudar a eliminar muchas de las condiciones que contribuyen al embarazo en adolescentes y a mitigar muchas de las consecuencias que sufren la niña, su hogar y su comunidad (p.28)

Lo anterior significa que, además de los derechos con que cuenta la adolescencia, las adolescentes madres merecen protecciones especiales que les garanticen bienestar y desarrollo social; en protecciones especiales, se entiende en este estudio, las sillas de la institución, los uniformes e, incluso, un cuarto destinado para la lactancia exclusivamente. Tales requerimientos representan una necesidad básica para la población de adolescentes madres dentro de los centros educativos del país, aunque la realidad es que casi en ningún centro educativo se cuenta con este espacio, a pesar de que la Viceministra Académica del Ministerio de Educación Pública informó que los centros educativos de todo el país deberán garantizar a las estudiantes espacios aptos para la lactancia durante la jornada lectiva en caso de que así se requiera (Gonzales, 2013).

En torno al tema, Calderón (2013) expresa que "el derecho a la lactancia materna y el amamantamiento del bebé no es una prerrogativa o una concesión antojadiza que queda al arbitrio de quienes administran el centro educativo; es un derecho humano fundamental que estamos llamados a garantizar plenamente". A pesar de lo anterior, este derecho es negado a las adolescentes madres, un factor que podría alejarlas de las aulas por la falta 


\section{.}

de espacios y medios necesarios para amamantar a sus hijos, lo cual se ve agravado por una escasa comunicación con la institución y apoyo familiar.

La falta de estos espacios para la lactancia materna nos enfrenta a la ambivalencia que se ha venido describiendo en el análisis acerca de las políticas y su manejo; en este caso, cabe mencionar la Política Pública de Lactancia Materna (2009), la cual tiene como objetivo maximizar la salud integral de las madres, niñas y niños, jóvenes, familias y población en general; por consiguiente, la falta de lactarios en los centros educativos evidencia una violación contra los derechos de las madres adolescentes, así como de la aplicación de la Política Pública de Lactancia Materna, cuyo acatamiento no ha pasado del papel, lo cual disminuye la posibilidad de un adecuado estado de salud tanto de la madre como del niño: en caso de la madre, debido a la preocupación de no poder amamantar a su hijo del todo por no contar con un espacio idóneo o por hacerlo en condiciones insalubres; en cuanto al infante, por no recibir la lactancia o, bien, recibirla en lugares inadecuados.

La existencia de estos espacios contribuye a fomentar dichos vínculos u se constituyen en una forma de incentivar el contenido de la Ley 7735 General de Protección a la Madre Adolescente (1997), que promueve la atención integral de las adolescentes, así como de acciones para el fomento de la maternidad y paternidad responsables, por ende, tales espacios no solo están garantizados por ley, sino que son un derecho innegable. Para las madres, es indispensable que se respete ese derecho, el contar con espacios para compartir con sus hijos, acción que a su vez implica el respeto de la Política Nacional para la Niñez y Adolescencia (2009) en la que se indica que el afecto es esencial para el desarrollo y la autodeterminación de los niños, niñas y adolescentes.

Se debe señalar que las mejoras no solo deben en cuanto a brindar el espacio físico, sino que debe existir una planeación integral que permita adecuar el tiempo adolescente madre con su bebé con el horario de clases. Por un lado, la adecuación del horario permitiría a las madres adolescentes permanecer dentro del sistema educativo, dado que la deserción es un hecho frecuente dentro de esta población, debido a que las jóvenes dejan de estudiar para cuidar a sus hijos, hijas, o para entrar en el campo laboral para poder, de esta forma, suplir las necesidades que tienen (UNFPA, 2013).

Para que estas condiciones se den dentro del centro educativo es importante que las jóvenes permanezcan activas, mas para lograrlo, de acuerdo con la UNFPA (2013), es necesario contar con el apoyo familiar y de sus parejas o los padres de sus hijos, dado que son uno de los principales factores protectores.

Respecto a la importancia del apoyo familiar, el UNFPA (2013) afirma que los padres desempeñan un papel fundamental en lo que se refiere al futuro de sus hijas, dado que pueden brindar información valiosa sobre ciertos temas, como la sexualidad, acerca del que también pueden incurrir en omisiones, lo cual trae repercusiones en la construcción de creencias en sus hijas. Al respecto, se menciona que los padres pueden ayudar a las niñas a desarrollar sus habilidades prácticas y fomentar que sean autónomas o bien pueden sucumbir ante las presiones económicas y de la comunidad y forzarlas a matrimonio y a una vida de dependencia" (ibíd., p. 50), afirmación que evidencia ese papel central de los progenitores en la construcción de la identidad y el futuro de sus hijas, debido a que su trato y apoyo influirán positiva y/o negativamente en la forma en que estas adolescentes se desenvuelvan en su vida. 


\section{.}

Lo anterior coincide con lo que declara la Ley de Paternidad Responsable (2001), en el Artículo 6 referente a políticas públicas donde, en el que se destaca que la crianza y todo lo referente al hijo o la hija es responsabilidad de ambos progenitores, es una responsabilidad y un derecho que ambos padres deben de compartir y cumplir de acuerdo con lo que dicta la política de protección integral de los derechos de las personas menores de edad. En el caso de que esta condición no ocurriera, y la adolescente madre no contara con el apoyo familiar, en el país, la Ley 7735 aporta acciones al respecto, en el Artículo 4 inciso g, en el cual se estipula "la construcción de albergues temporales, para las madres adolescentes que no cuenten con apoyo de sus familias" (p.2), de modo que las adolescentes madres que no tengan el apoyo de sus familiares deberían contar con un respaldo de ley para que se les debe garantice un lugar apto para que ellas y sus infantes se desenvuelvan en un lugar con condiciones adecuadas.

Tales planteamientos refuerzan la importancia de que tanto el gobierno, la sociedad en general, como las familias y las parejas de las adolescentes madres, continúen promoviendo oportunidades que les permitan continuar dentro del sistema educativo, objetivo más que relevante, puesto que alcanzar un alto grado académico aumenta las posibilidades de obtener una mejor calidad de vida en el futuro, además de que el deseo de estudiar resulta un factor protector en el que la Enfermería puede intervenir. Aunado a lo anterior, hay que recalcar la posición que adopta la Política Nacional para la Niñez y Adolescencia (2009) en la cual se menciona que "la educación es el medio idóneo para que las personas adquieran, desarrollen y potencien las capacidades y destrezas" (p.76), por tanto, la educación debe basarse en derechos humanos, educación y debe ser contextualizada e incluir la educación sexual, entre otras.

También es importante destacar que la posición de las adolescentes madres es contraria a lo establecido por el informe de la UNFPA (2013), ya que establece que el embarazo adolescente tiene entre sus efectos la interrupción o terminación de la educación formal, por la influencia de la sociedad que no les permite ni les brinda las oportunidades necesarias para que se mantengan dentro del centro educativo, lo cual implica la pérdida de oportunidades para desarrollar todo su potencial educativo, social y económico.

Las situaciones que viven actualmente las adolescentes madres necesitan de un insumo extra, como el del enfoque de las habilidades para la vida, definidas por Mangrulkar, Whitman y Posner (2001), como "las destrezas para permitir que los adolescentes adquieran las aptitudes necesarias para el desarrollo humano y para enfrentar en forma efectiva los retos de la vida diaria" (p.5); no obstante, no se trata solo de informar, sino de incluir las habilidades para la vida en los planes de atención y dirigir las intervenciones a partir de las políticas que resguardan los derechos de la población adolescente.

Además, es importante tener presentes las maneras de contrarrestar la problemática del embarazo adolescente propuestas por el UNFPA (2013) respecto de proteger los derechos humanos, fomentar la educación sexual y promover un desarrollo equitativo.

La Enfermería debe intervenir adecuadamente para promover acciones preventivas en salud, luchar por una mejor atención a nivel primario, disminuir la desigualdad de género, y fomentar el empoderamiento de la situación de derecho de las personas y, sobre todo, de su salud para que en las madres adolescentes no disminuya el grado de salud y de sus oportunidades de desarrollo social, puesto que solo de esta forma se garantizará un mejoramiento en la calidad de vida de estas personas quienes merecen vivir mejor. 

(1)

.

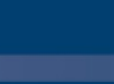

\section{Revista Electrónica Enfermeria Actual de costa Rica}

\section{CONCLUSIONES}

Dentro de sus proyectos de vida a corto plazo, las adolescentes entrevistadas consideran un hecho posible el continuar con sus estudios, deseo alentador, debido al coraje que muestran respecto de seguir adelante, evitando una futura deserción de centro educativo y que el bebé por nacer les brindara las fuerzas y la motivación personal para salir adelante y proveer a sus hijos e hijas las mejores oportunidades, como resultado del esfuerzo que conlleva el estudiar y ser madres a la vez.

En el tema de políticas y normativas, se colige que estas adolescentes madres no conocen la legislación que las ampara como adolescentes por un lado y, por otro lado, también como madres.

Se evidencia la necesidad de que las instituciones se involucren más a fondo con las adolescentes madres para conocer sus carencias dentro del ámbito educativo relacionado con su estado de embarazo y futura maternidad, las cuales, en este caso, podrían ser solucionadas por los encargados dentro del colegio respectivo.

Algunas facilidades como sillas más cómodas y contar con el permiso para utilizar blusas distintas a las del uniforme que se adapten cómodamente a la anatomía cambiante de las madres adolescentes, o proporcionar uniformes para el caso de una mujer embarazada, son consideradas por las entrevistadas como verdaderas necesidades que el colegio podría satisfacer con el fin de facilitar su estancia en el colegio y asegurar su permanencia en este.

Desde su intervención integral, Enfermería está en la capacidad de detectar las necesidades que posean las jóvenes madres y pueden valerse de elementos como el Proceso de Atención de Enfermería y las teorías de Enfermería para plantear soluciones en conjunto con las adolescentes y lograr resultados que convengan y mejoren su calidad de vida.

Por último, se evidencia la necesidad de brindar educación acerca de las políticas públicas, no solo en el hogar, sino también en los centros educativos para que así, cualquier ciudadano, en este caso las adolescentes madres, conozca sus derechos y los hagan valer como corresponde a partir del empoderamiento en salud. 


\section{Revista Electrónica Enfermeria Actual de costa Rica}

\section{REFERENCIAS BIBLIOGRAFICAS}

Anchía, S., Badilla, D., Cordero, A., Marín, K. (2012). Adolescentes embarazadas: Un análisis de la prestación de los servicios de Trabajo Social desde los Hospitales: México, San Juan de Dios, Dr. Rafael Ángel Calderón Guardia y Hospital de las Mujeres Dr. Adolfo Carit Eva, a la luz de la Política Social en Salud dirigida a esta población, durante los años 2011 y 2012. (Tesis de Licenciatura en Trabajo Social). Universidad de Costa Rica, Sede Rodrigo Facio.

Asamblea Legislativa de la República de Costa Rica. (2001). Ley de Paternidad Responsable y Registro Civil. Recuperado de http://www.oas.org/dil/esp/Ley Paternidad Responsable Costa Rica.pdf

Asamblea Legislativa de la República de Costa Rica. (1997). Ley General de Protección a la Madre Adolescente. Recuperado de http://cpj.go.cr/docs/derechos/madre-adolescente.pdf

Báez, J., Tudela, P. (2009). La fenomenología. Investigación cualitativa. (2 ${ }^{\text {da }}$ ed.). Madrid: ESIC Editorial.

Barrientos, L. y Tobar, V. (2011). Determinantes sociales de la salud relacionadas con el embarazo en la adolescencia en el área geográfica de responsabilidad de la unidad de salud la hachadura municipio de San Francisco Menéndez Departamento de Ahuachapán. Revista Universidad del Salvador. 146.

Calderón, D. (2013, 31 de julio). MEP reitera derecho a la lactancia en los centros educativos del país. mep.go.cr. Recuperado de http://www.mep.go.cr/noticias/mep-reitera-derecho-lactancia-centros-educativos-pais-0

Contreras, L. (2013, 11 de junio). Campaña pretende la prevención del embarazo en la adolescencia. crhoy.com. Recuperado de http://www.crhoy.com/campana-pretende-la-prevencion-del-embarazo-en-la-adolescencia/

García, J., Delval, J. (2010). Psicología del Desarrollo I. Madrid: UNED.

Gonzales, A. (2013, 5 de agosto). Centros de educación deben ofrecer espacios aptos para la lactancia según MEP. La Nación. Recuperado de http://www.nacion.com/nacional/Centros-educacion-espacios-lactanciaMEP 0 1358064345.html

Hernández, R., Fernández, C., Baptista, P. (2010). Metodología de la Investigación (5 ${ }^{\text {ta }}$ ed.). México D. F.: McGraw-Hill.

Krauskopof, D. (1999). El desarrollo psicológico en la adolescencia: las transformaciones en una época de cambios. Revista adolescencia y salud. 1 (2). 1.

López, Y. (2011) Embarazo en la adolescencia y su repercusión biopsicosocial sobre el organismo de la madre y su futuro hijo. Revista Cubana de Enfermería. Recuperado de http://scielo.sld.cu/scielo.php?script=sci arttext\&pid=S086403192011000400011 
Mangrulkar, L., Whitman, C., Posner, M. (2001). Enfoque de habilidades para la vida para un desarrollo saludable de niños $y$ adolescentes. [pdf]. Recuperado de http://conferenciapesmexico2012.com/memorias/wpcontent/themes/tema_memorias_confpes2012/downloads/Cursos/HABILIDADES_PARA LA_VIDA/Materiales_y_documentos/Enfoque\%20de\%20Habilidades\%20para\%201a\%20vida\%20OPS.pdf [Consulta 29 de setiembre de 2013].

Ministerio de Salud de la República de Costa Rica. (2009). Política Pública de Lactancia Materna. Recuperado de http://www.ministeriodesalud.go.cr/gestores_en_salud/lactancia/politica_lactancia_materna.pdf

Molina, M., Ferrada, C., Pérez, R., Cid, L., Casanueva, V. y García. (2004). Embarazo en la Adolescencia y su relación con la deserción escolar. Revista Médica de Chile. 132(1). 2-4.

Noguera, N. y Alvarado, H. (2012). Embarazo en la adolescencia. Una mirada desde el cuidado del Enfermería. Bogotá: Universidad del Bosque.

OMS (2013). Salud de los Adolescentes. Recuperado de http://www.who.int/topics/adolescent_health/es/

OPS (2008). La Renovación de la Atención Primaria de Salud en las Américas. Washington D.C.: OPS.

Páramo, M. (2009). Adolescencia y Psicoterapia: Análisis de significados a través de grupos de discusión. España: Ediciones Universidad Salamanca.

Quesada, M., Romero, M., Prieto, M., y Rodríguez, C. (2010). Caracterización social del embarazo en la adolescencia. Revista Archivo Médico Camagüey. Recuperado de http://scielo.sld.cu/scielo.php?pid=S1025$\underline{02552010000300010 \& \text { script }=\text { sci arttext }}$

Redondo, C., Galdó, G., y García, M. (2008). Atención al adolescente. Madrid: Ediciones de la Universidad de Cantabria.

Soutullo, C., Mardomingo, M. (2010). Manual de Psiquiatría del Niño y del Adolescente. Madrid: Editorial Panamericana.

UNESCO (2005). Declaración Universal sobre Bioética y Derechos Humanos. París: UNESCO.

UNFPA (2013). Maternidad en la niñez. Enfrentar el reto del embarazo en adolescentes. New York: ONU.

UNICEF (2009). Política Nacional para la Niñez y la Adolescencia. Recuperado de http://www.unicef.org/costarica/docs/cr_pub_Politica_NNA_CR.pdf

Zamora, A. (2011). Embarazo en niñas y adolescentes. Recuperado de www.ministeriodesalud.go.cr/.../1256-embarazo-enninas-y-adolescentes 